\title{
Consensus-Based Linear Distributed Filtering
}

\author{
Ion Matei ${ }^{\text {a }}$ John S. Baras ${ }^{b}$ \\ a Institute for Research in Electronics and Applied Physics, University of Maryland, College Park 20742, and Engineering Laboratory, \\ National Institute of Standards and Technology, Gaithersburg 20899 \\ ${ }^{\mathrm{b}}$ Institute for Systems Research and Department of Electrical and Computer Engineering, University of Maryland, College Park 20742
}

\begin{abstract}
We address the consensus-based distributed linear filtering problem, where a discrete time, linear stochastic process is observed by a network of sensors. We assume that the consensus weights are known and we first provide sufficient conditions under which the stochastic process is detectable, i.e. for a specific choice of consensus weights there exists a set of filtering gains such that the dynamics of the estimation errors (without noise) is asymptotically stable. Next, we develop a distributed, sub-optimal filtering scheme based on minimizing an upper bound on a quadratic filtering cost. In the stationary case, we provide sufficient conditions under which this scheme converges; conditions expressed in terms of the convergence properties of a set of coupled Riccati equations.
\end{abstract}

Key words: distributed filtering, consensus, sensor networks

\section{Introduction}

Sensor networks have broad applications in surveillance and monitoring of an environment, collaborative processing of information, and gathering scientific data from spatially distributed sources for environmental modeling and protection. A fundamental problem in sensor networks is developing distributed algorithms for state estimation of a process of interest. Generically, a process is observed by a group of (mobile) sensors organized in a network. The goal of each sensor is to compute accurate state estimates. The distributed filtering (estimation) problem has received a lot of attention during the past thirty years. An important contribution was made by Borkar and Varaiya [1], who address the distributed estimation problem of a random variable by a group of sensors. The particularity of their formulation is that both estimates and measurements are shared among neighboring sensors. The authors show that if the sensors form a communication ring, through which information is exchanged infinitely often, then the estimates converge asymptotically

\footnotetext{
^ This material is based upon work supported in part by the US Air Force Office of Scientific Research MURI award FA955009-1-0538, in part by the Defence Advanced Research Projects Agency (DARPA) under award number 013641-001 for the MultiScale Systems Center (MuSyC) through the FRCP of SRC and DARPA.

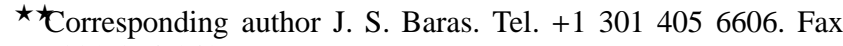
+13013148486.
}

Email addresses: imatei@umd.edu (Ion Matei), baras@umd.edu (John S. Baras). to the same value, i.e. they asymptotically agree. An extension of the results in reference [1] is given in [9]. The recent technological advances in mobile sensor networks have reignited the interest for the distributed estimation problem. Most papers focusing on distributed estimation propose different mechanisms for combining the Kalman filter with a consensus filter in order to ensure that the estimates asymptotically converge to the same value, schemes which will be henceforth called consensus-based distributed filtering (estimation) algorithms. In [7] and [6], several algorithms based on the idea mentioned above are introduced. In [2], the authors study the interaction between the consensus matrix, the number of messages exchanged per sampling time, and the Kalman gain for scalar systems. It is shown that optimizing the consensus matrix for fastest convergence and using the centralized optimal gain is not necessarily the optimal strategy if the number of exchanged messages per sampling time is small. In [8], the weights are adaptively updated to minimize the variance of the estimation error. Both the estimation and the parameter optimization are performed in a distributed manner. The authors derive an upper bound of the error variance in each node which decreases with the number of neighboring nodes.

In this note we address the consensus-based distributed linear filtering problem as well. We assume that each agent updates its (local) estimate in two steps. In the first step, an update is produced using a Luenberger observer type of filter. In the second step, called consensus step, every sensor computes a convex combination between its local update and the updates received from the neighboring sensors. 
Our focus is not on designing the consensus weights, but on designing the filter gains. For given consensus weights, we will first give sufficient conditions for the existence of filter gains such that the dynamics of the estimation errors (without noise) is asymptotically stable. These sufficient conditions are also expressible in terms of the feasibility of a set of linear matrix inequalities. Next, we present a distributed (in the sense that each sensor uses only information available within its neighborhood), sub-optimal filtering algorithm, valid for time varying topologies as well, resulting from minimizing an upper bound on a quadratic cost expressed in terms of the covariance matrices of the estimation errors. In the case where the matrices defining the stochastic process and the consensus weights are time invariant, we present sufficient conditions such that the aforementioned distributed algorithm produces filter gains which converge and ensure the stability of the dynamics of the covariance matrices of the estimation errors.

Paper structure: In Section II we describe the problems addressed in this paper. Section III introduces the sufficient conditions for detectability under the consensus-based linear filtering scheme together with a test expressed in terms of the feasibility of a set of linear matrix inequalities. In Section IV we present a sub-optimal distributed consensus based linear filtering scheme with quantifiable performance.

Notations and Abbreviations: We represent the property of positive definiteness (semi-definiteness) of a symmetric matrix $A$ by $A>0(A \geq 0)$. By convention, we say that a symmetric matrix $A$ is negative definite (semi-definite) if $-A>0(-A \geq 0)$ and we denote this by $A<0(A \leq 0)$. By $A>$ $B$ we understand that $A-B$ is positive definite. We use the abbreviations CBDLF for consensus-based linear filter(ing).

Remark 1 Given a positive integer $N$, a set of vectors $\left\{x_{i}\right\}_{i=1}^{N}$, a set of non-negative scalars $\left\{p_{i}\right\}_{i=1}^{N}$ summing up to one and a positive definite matrix $Q$, the following holds

$$
\left(\sum_{i=1}^{N} p_{i} x_{i}\right)^{\prime} Q\left(\sum_{i=1}^{N} p_{i} x_{i}\right) \leq \sum_{i=1}^{N} p_{i} x_{i}^{\prime} Q x_{i}
$$

Remark 2 Given a positive integer $N$, a set of vectors $\left\{x_{i}\right\}_{i=1}^{N}$, a set of matrices $\left\{A_{i}\right\}_{i=1}^{N}$ and a set of non-negative scalars $\left\{p_{i}\right\}_{i=1}^{N}$ summing up to one, the following holds

$$
\left(\sum_{i=1}^{N} p_{i} A_{i} x_{i}\right)\left(\sum_{i=1}^{N} p_{i} A_{i} x_{i}\right)^{\prime} \leq \sum_{i=1}^{N} p_{i} A_{i} x_{i} x_{i}^{\prime} A_{i}^{\prime}
$$

\section{Problem formulation}

We consider a stochastic process modeled by a discrete-time linear dynamic equation

$$
x(k+1)=A(k) x(k)+w(k), x(0)=x_{0},
$$

where $x(k) \in \mathbb{R}^{n}$ is the state vector and $w(k) \in \mathbb{R}^{n}$ is a driving noise, assumed Gaussian with zero mean and (possibly time varying) covariance matrix $\Sigma_{w}(k)$. The initial condition $x_{0}$ is assumed to be Gaussian with mean $\mu_{0}$ and covariance matrix $\Sigma_{0}$. The state of the process is observed by a network of $N$ sensors indexed by $i$, whose sensing models are given by

$$
y_{i}(k)=C_{i}(k) x(k)+v_{i}(k), i=1 \ldots N,
$$

where $y_{i}(k) \in \mathbb{R}^{r_{i}}$ is the observation made by sensor $i$ and $v_{i}(k) \in \mathbb{R}^{r_{i}}$ is the measurement noise, assumed Gaussian with zero mean and (possibly time varying) covariance matrix $\Sigma_{v_{i}}(k)$. We assume that the matrices $\left\{\Sigma_{v_{i}}(k)\right\}_{i=1}^{N}$ and $\Sigma_{w}(k)$ are positive definite for $k \geq 0$ and that the initial state $x_{0}$, the noises $v_{i}(k)$ and $w(k)$ are independent for all $k \geq 0$.

The set of sensors form a communication network whose topology is modeled by a directed graph that describes the information exchanged among agents. The goal of the agents is to (locally) compute estimates of the state of the process (1).

Let $\hat{x}_{i}(k)$ denote the state estimate computed by sensor $i$ at time $k$ and let $\epsilon_{i}(k)$ denote the estimation error, i.e. $\epsilon_{i}(k) \triangleq$ $x(k)-\hat{x}_{i}(k)$. The covariance matrix of the estimation error of sensor $i$ is denoted by $\Sigma_{i}(k) \triangleq E\left[\epsilon_{i}(k) \epsilon_{i}(k)^{\prime}\right]$, with $\Sigma_{i}(0)=\Sigma_{0}$.

The sensors update their estimates in two steps. In the first step, an intermediate estimate, denoted by $\varphi_{i}(k)$, is produced using a Luenberger observer filter

$$
\varphi_{i}(k)=A(k) \hat{x}_{i}(k)+L_{i}(k)\left(y_{i}(k)-C_{i}(k) \hat{x}_{i}(k)\right), i=1 \ldots N,
$$

where $L_{i}(k)$ is the filter gain.

In the second step, the new state estimate of sensor $i$ is generated by a convex combination between $\varphi_{i}(k)$ and all other intermediate estimates within its communication neighborhood, i.e.

$$
\hat{x}_{i}(k+1)=\sum_{j=1}^{N} p_{i j}(k) \varphi_{j}(k), i=1 \ldots N,
$$

where $p_{i j}(k)$ are non-negative scalars summing up to one ( $\left.\sum_{j=1}^{N} p_{i j}(k)=1\right)$, and $p_{i j}(k)=0$ if no link from $j$ to $i$ exists at time $k$. Having $p_{i j}(k)$ dependent on time accounts for a possibly time varying communication topology.

Remark 3 For notational simplicity, in what follows we will ignore the time dependence of the parameters of the model, i.e. the matrices $A(k), C_{i}(k), \Sigma_{w}(k), \Sigma_{v_{i}}(k)$ and the probabilities $p_{i j}(k)$.

Combining (3) and (4) we obtain the dynamic equations for the consensus based distributed filter:

$$
\hat{x}_{i}(k+1)=\sum_{j=1}^{N} p_{i j}\left[A \hat{x}_{j}(k)+L_{j}(k)\left(y_{j}(k)-C_{j} \hat{x}_{j}(k)\right)\right],
$$


for $i=1 \ldots N$. From (5) the estimation errors evolve according to

$$
\epsilon_{i}(k+1)=\sum_{j=1}^{N} p_{i j}\left[\left(A-L_{j}(k) C_{j}\right) \epsilon_{j}(k)+w(k)-L_{j}(k) v_{j}(k)\right] .
$$

Definition 4 (distributed detectability) Let the system (1)(2) together with $\boldsymbol{p}(k) \triangleq\left\{p_{i j}(k)\right\}_{i, j=1}^{N}$ be time invariant. We say that the linear process (1) is detectable using the CBDLF scheme (5), if there exist a set of matrices $\boldsymbol{L} \triangleq\left\{L_{i}\right\}_{i=1}^{N}$ such that the system (6), without the driving and measurement noises, is asymptotically stable, i.e. $\lim _{k \rightarrow \infty} \epsilon_{i}(k)=0$.

We introduce the following finite horizon quadratic filtering cost function

$$
J_{K}(\mathbf{L}(K))=\sum_{k=0}^{K} \sum_{i=1}^{N} E\left[\left\|\epsilon_{i}(k)\right\|^{2}\right]
$$

where by $\mathbf{L}(K)$ we understand the set of matrices $\mathbf{L}(K) \triangleq$ $\left\{L_{i}(k), k=0 \ldots K-1\right\}_{i=1}^{N}$. The optimal filtering gains represent the solution of the following optimization problem

$$
\mathbf{L}_{o}(K)=\arg \min _{\mathbf{L}(K)} J_{K}(\mathbf{L}(K))
$$

In the case the system (1)-(2) and the probabilities $\mathbf{p}(k) \triangleq$ $\left\{p_{i j}(k)\right\}_{i, j=1}^{N}$ are time invariant, we can also define the infinite horizon filtering cost function

$$
J_{\infty}(\mathbf{L})=\lim _{K \rightarrow \infty} \frac{1}{K} J_{K}(\mathbf{L})=\lim _{k \rightarrow \infty} \sum_{i=1}^{N} E\left[\left\|\epsilon_{i}(k)\right\|^{2}\right],
$$

where $\mathbf{L} \triangleq\left\{L_{i}\right\}_{i=1}^{N}$ is the set of steady state filtering gains. By solving the optimization problem

$$
\mathbf{L}_{o}=\arg \min _{\mathbf{L}} J^{\infty}(\mathbf{L}),
$$

we obtain the optimal steady-state filter gains.

In the next sections we will address the following problems:

Problem 5 (Detectability conditions) Under the above setup, we want to find conditions under which the system (1) is detectable in the sense of Definition 4.

Problem 6 (Sub-optimal scheme for consensus based distributed filtering) Ideally, we would like to obtain the optimal filter gains by solving the optimization problems (8) and (10), respectively. Due to the complexity and intractability of these problems, we will not provide the optimal filtering gains but rather focus on providing a sub-optimal scheme with quantifiable performance.

\section{Distributed detectability}

In this section we give sufficient conditions under which the (time-invariant) system (1) is detectable in the sense of Definition 4 and provide a detectability test in terms of the feasibility of a set of LMIs. We start with a result that motivates the intuition behind combining the consensus step with the Luenberger observer for performing distributed filtering.

Proposition 7 Consider the linear time-invariant dynamics (1)-(2). Assume that in the CBDLF scheme (5), we have $p_{i j}=\frac{1}{N}$ and that $\hat{x}_{i}(0)=\hat{x}_{0}$, for all $i, j=1 \ldots N$. If the pair $(A, C)$ is detectable, where $C^{\prime}=\left[C_{1}^{\prime} \ldots C_{N}^{\prime}\right]^{\prime}$, then the system (1)-(2) is detectable as well, in the sense of Definition 4.

PROOF. Under the assumption that $p_{i j}=\frac{1}{N}$ and $\hat{x}_{i}=x_{0}$ for all $i, j=1 \ldots N$, it follows that the estimation errors respect the dynamics

$$
\epsilon(k+1)=\frac{1}{N} \sum_{i=1}^{N}\left(A-L_{i} C_{i}\right) \epsilon(k)=\left(A-\frac{1}{N} L C\right) \epsilon(k)
$$

where $L=\left[L_{1}, L_{2}, \ldots, L_{N}\right]$.

Since the pair $(A, C)$ is detectable, there exists a matrix $L^{*}=\left[L_{1}^{*}, L_{2}^{*}, \ldots, L_{N}^{*}\right]$ such that $A-\frac{1}{N} L^{*} C$ has all eigenvalues within the unit circle and therefore the dynamics (11) is asymptotically stable, which implies that (1) is detectable in the sense of Definition 4.

The previous proposition tells us that if we achieve (average) consensus between the state estimates at each time instant, and if the pair $(A, C)$ is detectable (in the classical sense), then the system (1) is detectable in the sense of Definition 4. However, achieving consensus at each time instant can be costly in both time and numerical complexity. In addition, it turns out that using consensus for collaboration does not guarantee stability of the estimation errors, even in the case where the estimation errors, without collaboration, are stable. For example, in the system (1)-(2), let

$$
A=\left(\begin{array}{cc}
1 & 1.5 \\
0.2 & 2
\end{array}\right), C_{1}=\left(\begin{array}{ll}
1 & 0 \\
0 & 1
\end{array}\right), C_{2}=\left(\begin{array}{ll}
1 & 2 \\
2 & 1
\end{array}\right)
$$

Two locally stabilizing filtering gains are

$$
L_{1}=\left(\begin{array}{cc}
1 & -0.5 \\
0.2 & 1.5
\end{array}\right), L_{2}=\left(\begin{array}{cc}
0.8333 & -0.1667 \\
1.9333 & -1.8667
\end{array}\right)
$$

It can be checked that both $A-L_{1} C_{1}$ and $A-L_{2} C_{2}$ have stable eigenvalues, and therefore the system is detectable when there is no collaboration. However, if the two sensors do collaborate, using as consensus weights $p_{11}=p_{12}=p_{21}=$ $p_{22}=0.5$, it can be checked that (6) (without the noise) is 
unstable. Therefore, it is of interest to derive (testable) conditions under which the CBDLF produces stable estimation errors (in the mean square sense).

Lemma 8 (sufficient conditions for distributed detectability) If there exists a set of symmetric, positive definite matrices $\left\{Q_{i}\right\}_{i=1}^{N}$ and a set of matrices $\left\{L_{i}\right\}_{i=1}^{N}$ such that

$$
Q_{i}=\sum_{j=1}^{N} p_{j i}\left(A-L_{j} C_{j}\right)^{\prime} Q_{j}\left(A-L_{j} C_{j}\right)+S_{i}, i=1 \ldots N
$$

for some positive definite matrices $\left\{S_{i}\right\}_{i=1}^{N}$, then the system (1) is detectable in the sense of Definition 4.

PROOF. The dynamics of the estimation error without noise is given by

$$
\epsilon_{i}(k+1)=\sum_{j=1}^{N} p_{i j}\left(A-L_{j} C_{j}\right) \epsilon_{j}(k), i=1 \ldots N .
$$

In order to prove the stated result we have to show that (13) is asymptotically stable. We define the Lyapunov function

$$
V(k)=\sum_{i}^{N} \epsilon_{i}(k)^{\prime} Q_{i} \epsilon_{i}(k)
$$

and our goal is to show that $V(k+1)-V(k)<0$ for all $k \geq 0$. The Lyapunov difference is given by

$$
\begin{gathered}
V(k+1)-V(k)=\sum_{i=1}^{N}\left(\sum_{j=1}^{N} p_{i j}\left(A-L_{j} C_{j}\right) \epsilon_{j}(k)\right)^{\prime} Q_{i} . \\
\cdot\left(\sum_{j=1}^{N} p_{i j}\left(A-L_{j} C_{j}\right) \epsilon_{j}(k)\right)-\epsilon_{i}(k)^{\prime} Q_{i} \epsilon_{i}(k) \leq
\end{gathered}
$$

$\leq \sum_{i=1}^{N}\left(\sum_{j=1}^{N} p_{i j} \epsilon_{j}(k)^{\prime}\left(A-L_{j} C_{j}\right)^{\prime} Q_{i}\left(A-L_{j} C_{j}\right) \epsilon_{j}(k)\right)-\epsilon_{i}(k)^{\prime} Q_{i} \epsilon_{i}(k)$,

where the inequality followed from Remark 1 . By changing the summation order we can further write

$$
\begin{gathered}
V(k+1)-V(k) \leq \sum_{i=1}^{N} \epsilon_{i}(k)^{\prime}\left(\sum_{j=1}^{N} p_{j i}\left(A-L_{j} C_{j}\right)^{\prime} Q_{j}\right. \\
\left.\cdot\left(A-L_{j} C_{j}\right)-Q_{i}\right) \epsilon_{i}(k) \leq-\sum_{i=1}^{N} \epsilon_{i}(k)^{\prime} S_{i} \epsilon_{i}(k)
\end{gathered}
$$

where the last inequality follows from (12). From the fact that $\left\{S_{j}\right\}_{j=1}^{N}$ are positive definite matrices, we get

$$
V(k+1)-V(k)<0,
$$

which implies that (13) is asymptotically stable.

The following result relates the existence of the sets of matrices $\left\{Q_{i}\right\}_{i=1}^{N}$ and $\left\{L_{i}\right\}_{i=1}^{N}$ such that (12) is satisfied, with the feasibility of a set of linear matrix inequalities (LMIs).
Proposition 9 (distributed detectability test) The linear system (1) is detectable in the sense of Definition 4 if the following linear matrix inequalities, in the variables $\left\{X_{i}\right\}_{i=1}^{N}$ and $\left\{Y_{i}\right\}_{i=1}^{N}$, are feasible

$\left(\begin{array}{cccc}X_{i} & \sqrt{p_{1 i}}\left(A^{\prime} X_{1}-C_{1}^{\prime} Y_{1}^{\prime}\right) & \cdots & \sqrt{p_{N i}}\left(A^{\prime} X_{N}-C_{N}^{\prime} Y_{N}^{\prime}\right) \\ \sqrt{p_{1 i}}\left(X_{1} A-Y_{1} C 1\right) & X_{1} & \cdots & 0 \\ \vdots & \vdots & \ddots & \vdots \\ \sqrt{p_{N i}}\left(X_{N} A-Y_{N} C_{N}\right) & 0 & \cdots & X_{N}\end{array}\right)>0$,

for $i=1 \ldots N$ and where $\left\{X_{i}\right\}_{i=1}^{N}$ are symmetric. Moreover, a stable CBDLF is obtained by choosing the filter gains as $L_{i}=X_{i}^{-1} Y_{i}$ for $i=1 \ldots N$.

PROOF. First we note that, by the Schur complements Lemma, the linear matrix inequalities (14) are feasible if and only if there exist a set a symmetric matrices $\left\{X_{i}\right\}_{i=1}^{N}$ and a set of matrices $\left\{Y_{i}\right\}_{i=1}^{N}$, such that

$$
X_{i}-\sum_{j=1}^{N} p_{j i}\left(X_{j} A-Y_{j} C_{j}\right)^{\prime} X_{j}^{-1}\left(X_{j} A-Y_{j} C_{j}\right)>0, X_{i}>0
$$

for all $i=1 \ldots N$. We further have that,

$$
X_{i}-\sum_{j=1}^{N} p_{j i}\left(A-X_{j}^{-1} Y_{j} C_{j}\right)^{\prime} X_{j}\left(X_{j} A-X_{j}^{-1} Y_{j} C_{j}\right)>0, X_{i}>0 .
$$

By defining $L_{i} \triangleq X_{i}^{-1} Y_{i}$, it follows that

$$
X_{i}-\sum_{j=1}^{N} p_{j i}\left(A-L_{j} C_{j}\right)^{\prime} X_{j}\left(A-L_{j} C_{j}\right)>0, X_{i}>0 .
$$

Therefore, if the matrix inequalities (14) are feasible, there exists a set of positive definite matrices $\left\{X_{i}\right\}_{i=1}^{N}$ and a set of positive matrices $\left\{S_{i}\right\}_{i=1}^{N}$, such that

$$
X_{i}=\sum_{j=1}^{N} p_{j i}\left(A-L_{j} C_{j}\right)^{\prime} X_{j}\left(A-L_{j} C_{j}\right)+S_{i}
$$

By Lemma 8, it follows that the linear dynamics (6), without noise, is asymptotically stable, and therefore the system (1)(2) is detectable in the sense of Definition 4.

\section{Sub-Optimal Consensus-Based Distributed linear Filtering}

Obtaining the closed form solution of the optimization problem (8) is a challenging problem, which is in the same spirit as the decentralized optimal control problem. In this section 
we provide a sub-optimal algorithm for computing the filter gains of the CBDLF with quantifiable performance, i.e. we compute a set of filtering gains which guarantee a certain level of performance with respect to the quadratic cost (7).

\subsection{Finite Horizon Sub-Optimal Consensus-Based Dis- tributed Linear Filtering}

The sub-optimal scheme for computing the CBDLF gains results from minimizing an upper bound of the quadratic filtering cost (7). The following proposition gives upperbounds for the covariance matrices of the estimation errors.

Lemma 10 Consider the following coupled difference equations

$$
\begin{gathered}
Q_{i}(k+1)=\sum_{i=1}^{N} p_{i j}\left[\left(A-L_{j}(k) C_{j}\right) Q_{j}(k)\left(A-L_{j}(k) C_{j}\right)^{\prime}+\right. \\
\left.+L_{j}(k) \Sigma_{v_{j}} L_{j}(k)^{\prime}\right]+\Sigma_{w},
\end{gathered}
$$

with $Q_{i}(0)=\Sigma_{i}(0)$, for $i=1 \ldots N$. The following inequality holds

$$
\Sigma_{i}(k) \leq Q_{i}(k),
$$

for $i=1 \ldots N$ and for all $k \geq 0$, where $\Sigma_{i}(k)$ is the covariance matrix of the estimation error of sensor $i$.

PROOF. Using (6), the matrix $\Sigma_{i}(k+1)$ can be explicitly written as

$$
\begin{gathered}
\sum_{i}(k+1)=E\left[\left(\sum_{j=1}^{N} p_{i j}\left(A-L_{j}(k) C_{j}\right) \epsilon_{j}(k)+w(k)-\right.\right. \\
\left.-\sum_{j=1}^{N} p_{i j} L_{j}(k) v_{j}(k)\right)^{\prime}\left(\sum_{j=1}^{N} p_{i j}\left(A-L_{j}(k) C_{j}\right) \epsilon_{j}(k)+w(k)-\right. \\
\left.\left.-\sum_{j=1}^{N} p_{i j}(k) L_{j}(k) v_{j}(k)\right)\right] .
\end{gathered}
$$

Using the fact that the noises $w(k)$ and $v_{i}(k)$ have zero mean, and they are independent with respect to themselves and $x_{0}$, for every time instant, we can further write

$$
\begin{gathered}
\Sigma_{i}(k+1)=E\left[( \sum _ { j = 1 } ^ { N } p _ { i j } ( A - L _ { j } ( k ) C _ { j } ) \epsilon _ { j } ( k ) ) ^ { \prime } \left(\sum_{j=1}^{N} p_{i j}(A-\right.\right. \\
\left.\left.\left.-L_{j}(k) C_{j}\right) \epsilon_{j}(k)\right)\right]+E\left[\left(\sum_{j=1}^{N} p_{i j} L_{j}(k) v_{j}(k)\right)^{\prime}\right. \\
\left.\left(\sum_{j=1}^{N} p_{i j} L_{j}(k) v_{j}(k)\right)\right]+\Sigma_{w} .
\end{gathered}
$$

By Remark 2, it follows that

$$
\begin{gathered}
E\left[\left(\sum_{j=1}^{N} p_{i j}\left(A-L_{j}(k) C_{j}\right) \epsilon_{j}(k)\right)^{\prime}\left(\sum_{j=1}^{N} p_{i j}\left(A-L_{j}(k) C_{j}\right) \epsilon_{j}(k)\right)\right] \\
\leq \sum_{j=1}^{N} p_{i j}\left(A-L_{j}(k) C_{j}\right) \Sigma_{j}(k)\left(A-L_{j}(k) C_{j}\right)^{\prime}
\end{gathered}
$$

and

$$
\begin{gathered}
E\left[\left(\sum_{j=1}^{N} p_{i j} L_{j}(k) v_{j}(k)\right)^{\prime}\left(\sum_{j=1}^{N} p_{i j} L_{j}(k) v_{j}(k)\right)\right] \preceq \\
\leq \sum_{j=1}^{N} p_{i j} L_{j}(k) \Sigma_{v_{j}} L_{j}(k)^{\prime}, i=1 \ldots N .
\end{gathered}
$$

From the previous two expressions, we obtain that

$$
\begin{gathered}
\Sigma_{i}(k+1) \leq \sum_{j=1}^{N} p_{i j}\left(A-L_{j}(k) C_{j}\right) \Sigma_{j}(k)\left(A-L_{j}(k) C_{j}\right)^{\prime}+ \\
+\sum_{j=1}^{N} p_{i j} L_{j}(k) \Sigma_{v_{j}} L_{j}(k)+\Sigma_{w}
\end{gathered}
$$

We prove (16) by induction. Assume that $\Sigma_{i}(k) \leq Q_{i}(k)$ for all $i=1 \ldots N$. Then

$$
\begin{aligned}
& \left(A-L_{i}(k) C_{i}\right) \Sigma_{i}(k)\left(A-L_{i}(k) C_{i}\right)^{\prime} \leq \\
\leq & \left(A-L_{i}(k) C_{i}\right) Q_{i}(k)\left(A-L_{i}(k) C_{i}\right)^{\prime},
\end{aligned}
$$

and

$$
L_{i}(k) \Sigma_{i}(k) L_{i}(k)^{\prime} \leq L_{i}(k) Q_{i}(k) L_{i}(k)^{\prime}, i=1 \ldots N .
$$

and therefore

$$
\Sigma_{i}(k+1) \preceq Q_{i}(k+1), i=1 \ldots N
$$

Defining the finite horizon quadratic cost function

$$
\bar{J}_{K}(\mathbf{L}(K))=\sum_{k=1}^{K} \sum_{i=1}^{N} \operatorname{tr}\left(Q_{i}(k)\right)
$$

the next Corollary follows immediately.

Corollary 11 The following inequalities hold

$$
J_{K}(\boldsymbol{L}(K)) \leq \bar{J}_{K}(\boldsymbol{L}(K)),
$$

and

$$
\limsup _{K \rightarrow \infty} \frac{1}{K} J_{K}(\boldsymbol{L}) \leq \limsup _{K \rightarrow \infty} \frac{1}{K} \bar{J}_{K}(\boldsymbol{L})
$$

PROOF. Follows immediately from Lemma 10.

In the previous Corollary we obtained an upper bound on the filtering cost function. Our sub-optimal consensus based distributed filtering scheme will result from minimizing this upper bound in terms of the filtering gains $\left\{L_{i}(k)\right\}_{i=1}^{N}$ :

$$
\min _{\mathbf{L}(K)} \bar{J}_{K}(\mathbf{L}(K))
$$

Proposition 12 The optimal solution for the optimization problem (20) is

$$
L_{i}^{*}(k)=A Q_{i}^{*}(k) C_{i}^{\prime}\left[\Sigma_{v_{i}}+C_{i} Q_{i}^{*}(k) C_{i}^{\prime}\right]^{-1},
$$

and the optimal value is given by

$$
\bar{J}_{K}^{*}\left(\boldsymbol{L}^{*}(K)\right)=\sum_{k=1}^{K} \sum_{i=1}^{N} \operatorname{tr}\left(Q_{i}^{*}(k)\right),
$$


where $Q_{i}^{*}(k)$ is computed using

$$
\begin{gathered}
Q_{i}^{*}(k+1)=\sum_{j=1}^{N} p_{i j}\left[A Q_{j}^{*}(k) A^{\prime}+\Sigma_{w}-A Q_{j}^{*}(k) C_{j}^{\prime}\right. \\
\left.\cdot\left(\Sigma_{v_{j}}+C_{j} Q_{j}^{*}(k) C_{j}^{\prime}\right)^{-1} C_{j} Q_{j}^{*}(k) A^{\prime}\right]
\end{gathered}
$$

with $Q_{i}^{*}(0)=\Sigma_{i}(0)$ and for $i=1 \ldots N$.

PROOF. Let $\bar{J}_{K}(\mathbf{L}(K))$ be the cost function when an arbitrary set of filtering gains $\mathbf{L}(K) \triangleq\left\{L_{i}(k), k=0 \ldots K-1\right\}_{i=1}^{N}$ is used in (15). We will show that $\bar{J}_{K}^{*}\left(\mathbf{L}^{*}(K)\right) \leq \bar{J}_{K}(\mathbf{L}(K))$, which in turn will show that $\mathbf{L}^{*}(K) \triangleq\left\{L_{i}(k)^{*}, k=0 \ldots K-\right.$ $1\}_{i=1}^{N}$ is the optimal solution of the optimization problem (20). Let $\left\{Q_{i}^{*}(k)\right\}_{i=1}^{N}$ and $\left\{Q_{i}(k)\right\}_{i=1}^{N}$ be the matrices obtained when $\mathbf{L}^{*}(K)$ and $\mathbf{L}(K)$, respectively are substituted in (15). In what follows we will show by induction that $Q_{i}^{*}(k) \preceq$ $Q_{i}(k)$ for $k \geq 0$ and $i=1 \ldots N$, which basically proves that $\bar{J}_{K}^{*}\left(\mathbf{L}^{*}(K)\right) \leq \bar{J}_{K}(\mathbf{L}(K))$, for any $\mathbf{L}(K)$. For simplifying the proof, we will omit in what follows the time index for some matrices and for the consensus weights.

Substituting $\left\{L_{i}^{*}(k), k \geq 0\right\}_{i=1}^{N}$ in (15), after some matrix manipulations we get

$$
\begin{aligned}
& Q_{i}^{*}(k+1)=\sum_{j=1}^{N} p_{i j}\left[A Q_{j}^{*}(k) A^{\prime}+\Sigma_{w}-A Q_{j}^{*}(k) C_{j}^{\prime}\left(\Sigma_{v_{j}}+\right.\right. \\
& \left.\left.+C_{j} Q_{j}^{*}(k) C_{j}^{\prime}\right)^{-1} C_{j} Q_{j}^{*}(k) A^{\prime}\right], Q_{i}^{*}(0)=\Sigma_{i}(0), i=1 \ldots N .
\end{aligned}
$$

We can derive the following matrix identity:

$$
\begin{gathered}
\left(A-L_{i} C_{i}\right) Q_{i}\left(A_{i}-L_{i} C_{i}\right)^{\prime}+L_{i} \Sigma_{v_{i}} L_{i}^{\prime}=\left(A-L_{i}^{*} C_{i}\right) Q_{i}\left(A_{i}-L_{i}^{*} C_{i}\right)^{\prime}+ \\
+L_{i}^{*} \Sigma_{v_{i}} L_{i}^{* \prime}+\left(L_{i}-L_{i}^{*}\right)\left(\Sigma_{v_{i}}+C_{i} Q_{i} C_{i}^{\prime}\right)\left(L_{i}-L_{i}^{*}\right)^{\prime} .
\end{gathered}
$$

Assume that $Q_{i}^{*}(k) \leq Q_{i}(k)$ for $i=1 \ldots N$. Using identity (23), the dynamics of $Q_{i}(k)^{*}$ becomes

$$
\begin{gathered}
Q_{i}^{*}(k+1)=\sum_{j=1}^{N} p_{i j}\left(\left(A-L_{j}(k) C_{j}\right) Q_{j}(k)\left(A-L_{j}(k) C_{j}\right)^{\prime}+\right. \\
+L_{j}(k) \Sigma_{v_{j}} L_{j}(k)^{\prime}-\left(L_{j}(k)-L_{j}^{*}(k)\right)\left(\Sigma_{v_{j}}+C_{j} Q_{j}(k) C_{j}^{\prime}\right) . \\
\left.\cdot\left(L_{j}(k)-L_{j}^{*}(k)\right)^{\prime}+\Sigma_{w}\right) .
\end{gathered}
$$

The difference $Q_{i}^{*}(k+1)-Q_{i}(k+1)$ can be written as

$$
\begin{gathered}
Q_{i}(k+1)^{*}-Q_{i}(k+1)= \\
=\sum_{j=1}^{N} p_{i j}\left(\left(A-L_{j}(k) C_{j}\right)\left(Q_{j}^{*}(k)-Q_{j}(k)\right)\left(A-L_{j}(k) C_{j}\right)^{\prime}-\right. \\
\left.-\left(L_{j}(k)-L_{j}^{*}(k)\right)\left(\Sigma_{v_{j}}+C_{j} Q_{j}(k) C_{j}^{\prime}\right)\left(L_{j}(k)-L_{j}^{*}(k)\right)^{\prime}\right) .
\end{gathered}
$$

Since $\Sigma_{v_{i}}+C_{i} Q_{i}(k) C_{i}^{\prime}$ is positive definite for all $k \geq 0$ and $i=$ $1 \ldots N$, and since we assumed that $Q_{i}^{*}(k) \leq Q_{i}(k)$, it follows that $Q_{i}^{*}(k+1) \leq Q_{i}(k+1)$. Hence we obtained that

$$
\bar{J}_{K}^{*}\left(\mathbf{L}^{*}(K)\right) \leq \bar{J}_{K}(\mathbf{L}(K)),
$$

for any set of filtering gains $\mathbf{L}(K)=\left\{L_{i}(k), k=0 \ldots K-1\right\}_{i=1}^{N}$, which concludes the proof.
Since Proposition 12 holds for arbitrarily large values of $K$, we summarize in the following algorithm the sub-optimal CBDLF scheme.

\section{Algorithm 1}

1. Initialization: $\hat{x}_{i}(0)=\mu_{0}, Q_{i}(0)=\Sigma_{0}$

2. while new data exists

3. Compute the filter gains

$$
L_{i} \leftarrow A Q_{i} C_{i}^{\prime}\left(\Sigma_{v_{i}}+C_{i} Q_{i} C_{i}^{\prime}\right)^{-1}
$$

4. Update the state estimates:

$$
\begin{gathered}
\varphi_{i} \leftarrow A \hat{x}_{i}+L_{i}\left(y_{i}-C_{i} \hat{x}_{i}\right) \\
\hat{x}_{i} \leftarrow \sum_{j} p_{i j} \varphi_{j}
\end{gathered}
$$

5. Update the matrices $Q_{i}$ :

$$
Q_{i} \leftarrow \sum_{j=1}^{N} p_{i j}\left(\left(A-L_{j} C_{j}\right) Q_{j}\left(A-L_{j} C_{j}\right)^{\prime}+L_{j} \Sigma_{v_{j}} L_{j}^{\prime}\right)+\Sigma_{w}
$$

Note that the above algorithm does accommodate time varying systems and time varying topologies since the previous results do hold in the case where the matrices of the system and the probabilities $p_{i j}(k)$ are time varying, and can be implemented in a distributed manner, i.e., the agents use only information from their neighbors.

\subsection{Infinite Horizon Consensus Based Distributed Filter- ing}

We now assume that the matrices $A(k),\left\{C_{i}(k)\right\}_{i=1}^{N},\left\{\Sigma_{v_{i}}(k)\right\}_{i=1}^{N}$ and $\Sigma_{w}(k)$ and the weights $\left\{p_{i j}(k)_{i, j=1}^{N}\right\}$ are time invariant. We are interested in finding out under what conditions Algorithm 1 converges and if the filtering gains are stabilizing. From the previous section we note that the optimal infinite horizon cost can be written as

$$
\bar{J}_{\infty}^{*}=\lim _{k \rightarrow \infty} \sum_{i=1}^{N} \operatorname{tr}\left(Q_{i}^{*}(k)\right)
$$

where the dynamics of $Q_{i}(k)^{*}$ is given by

$$
\begin{gathered}
Q_{i}^{*}(k+1)=\sum_{j=1}^{N} p_{i j}\left[A Q_{j}^{*}(k) A^{\prime}+\Sigma_{w}-\right. \\
\left.-A Q_{j}^{*}(k) C_{j}^{\prime}\left(\Sigma_{v_{j}}+C_{j} Q_{j}^{*}(k) C_{j}^{\prime}\right)^{-1} C_{j} Q_{j}^{*}(k) A^{\prime}\right],
\end{gathered}
$$

and the optimal filtering gains are given by

$$
L_{i}^{*}(k)=A Q_{i}^{*}(k) C_{i}^{\prime}\left[\Sigma_{v_{i}}+C_{i} Q_{i}^{*}(k) C_{i}^{\prime}\right]^{-1},
$$


for $i=1 \ldots N$. Assuming that (24), converges, the optimal value of the cost $\bar{J}_{\infty}^{*}$ is given by

$$
\bar{J}_{\infty}^{*}=\sum_{i=1}^{N} \operatorname{tr}\left(\bar{Q}_{i}\right)
$$

where $\left\{\bar{Q}_{i}\right\}_{i=1}^{N}$ satisfy

$$
\bar{Q}_{i}=\sum_{j=1}^{N} p_{i j}\left[A \bar{Q}_{j} A^{\prime}+\Sigma_{w}-A \bar{Q}_{j} C_{j}^{\prime}\left(\Sigma_{v_{j}}+C_{j} \bar{Q}_{j} C_{j}^{\prime}\right)^{-1} C_{j} \bar{Q}_{j} A^{\prime}\right] .
$$

Sufficient conditions under which there exists a unique solution of (25) are provided by Proposition 16 (in the Appendix section), which says that if $(\mathbf{p}, \mathbf{L}, \mathbf{A})$ is detectable and $\left(\mathbf{A}, \Sigma_{v}^{1 / 2}, \mathbf{p}\right)$ is stabilizable in the sense of Definitions 13 and 14 , respectively, then there is a unique solution of (25) and $\lim _{k \rightarrow \infty} Q_{i}^{*}(k)=\bar{Q}_{i}$.

\section{References}

[1] V. Borkar and P. Varaya. Asymptotic agreement in distributed estimation. IEEE Trans. Autom. Control, AC-27(3):650-655, Jun 1982.

[2] R. Carli, A. Chiuso, L. Schenato, and S. Zampieri. Distributed kalman filtering based on consensus strategies. IEEE Journal on Selected Area in Communication, 26(4):622-633, May 2008.

[3] O.L.V. Costa and M.D. Fragoso. Stability results for discrete-time linear systems with markovian jumping parameters. Journal of Mathematical Analysis and Application, 179:154-178, 1993.

[4] O.L.V. Costa and M.D. Fragoso. Discrete-time coupled riccati equations for systems with markov switching parameters. Journal of Mathematical Analysis and Application, 194:197-216, 1995.

[5] O.L.V. Costa, M.D. Fragoso, and R.P. Marques. Discrete-Time Markov Jump Linear Systems. Springer-Verlag, London, 2005.

[6] R.O. Saber. Distributed kalman filter with embedded consensus filters. Proceedings of the 44th IEEE Conference on Decision and Control, 2005.

[7] R.O. Saber. Distributed kalman filtering for sensor networks. Proceedings of the 46th IEEE Conference on Decision and Control, pages 5492-5498, 2007.

[8] A. Speranzon, C. Fischione, K. H. Johansson, and A. SangiovanniVincentelli. A distributed minimum variance estimator for sensor networks. IEEE Journal on Select Areas in Communication, 26(4):609-621, May 2008.

[9] D. Teneketzis and P. Varaiya. Consensus in distributed estimation. Advances in Statistical Signal Processing, pages 361-386, Jan 1988.

\section{A Convergence of discrete-time coupled Riccati dy- namic equations}

Given a positive integer $N$, a sequence of positive numbers $\mathbf{p}=\left\{p_{i j}\right\}_{i, j=1}^{N}$ and a set of matrices $\mathbf{F}=\left\{F_{i}\right\}_{i=1}^{N}$, we consider the following matrix difference equations

$$
W_{i}(k+1)=\sum_{j=1}^{N} p_{i j} F_{j} W_{j}(k) F_{j}^{\prime}, W_{i}(0)=W_{i}^{0}, i=1 \ldots N
$$

Related to the above dynamic equations, we introduce the following stabilizability and detectability definitions.

Definition 13 [4] Given a set of matrices $C=\left\{C_{i}\right\}_{i=1}^{N}$, we say that $(\boldsymbol{p}, \boldsymbol{L}, \boldsymbol{A})$ is detectable if there exists a set of matrices $\boldsymbol{L}=\left\{L_{i}\right\}_{i=1}^{N}$ such that the dynamics (A.1) is asymptotically stable, where $F_{i}=A_{i}-L_{i} C_{i}$, for $i=1 \ldots N$.

Definition 14 [4] Given a set of matrices $C=\left\{C_{i}\right\}_{i=1}^{N}$, we say that $(\boldsymbol{A}, \boldsymbol{L}, \boldsymbol{p})$ is stabilizable, if there exists a set of matrices $\boldsymbol{L}=\left\{L_{i}\right\}_{i=1}^{N}$ such that the dynamics $(A .1)$ is asymptotically stable, where $F_{i}=A_{i}-C_{i} L_{i}$, for $i=1 \ldots N$.

Remark 15 In the same spirit of Proposition 9, numerical tests for checking the detectability and stabilizability properties, in the sense of the above definitions, can be expressed in terms of the feasibility of a set of LMIs. For more details, the interested reader can consult [3-5].

Consider the following coupled Riccati difference equations

$$
\begin{gathered}
Q_{i}(k+1)=\sum_{i=1}^{N} p_{i j}\left(A_{j} Q_{j}(k) A_{j}^{\prime}-A_{j} Q_{j}(k) C_{j}^{\prime}\left(C_{j} Q_{j}(k) C_{j}^{\prime}+\right.\right. \\
\left.\left.+\Sigma_{v_{j}}\right)^{-1} C_{j} Q_{j}(k) A_{j}^{\prime}+\Sigma_{w}\right), Q_{i}(0)=Q_{i}^{0}>0
\end{gathered}
$$

for $i=1 \ldots N$, where $\left\{\Sigma_{v_{i}}\right\}_{i=1}^{N}$ and $\Sigma_{w}$ are symmetric positive definite matrices.

Proposition 16 Let $\Sigma_{v}^{1 / 2}=\left\{\Sigma_{v_{i}}^{1 / 2}\right\}_{i=1}^{N}$, where $\Sigma_{v_{i}}=\Sigma_{v_{i}}^{1 / 2^{\prime}} \Sigma_{v_{i}}^{1 / 2}$. Suppose that $(\boldsymbol{p}, \boldsymbol{C}, \boldsymbol{A})$ is detectable and that $\left(\boldsymbol{A}, \Sigma_{v}^{1 / 2}, \boldsymbol{p}\right)$ is stabilizable in the sense of Definitions 13 and 14, respectively. Then there exists a unique set of symmetric positive definite matrices $\bar{Q}=\left\{\bar{Q}_{i}\right\}_{i=1}^{N}$ satisfying

$\bar{Q}_{i}=\sum_{i=1}^{N} p_{i j}\left(A_{j} \bar{Q}_{j} A_{j}^{\prime}-A_{j} \bar{Q}_{j} C_{j}^{\prime}\left(C_{j} \bar{Q}_{j} C_{j}^{\prime}+\Sigma_{v_{j}}\right)^{-1} C_{j} \bar{Q}_{j} A_{j}^{\prime}+\Sigma_{w}\right)$,

for $i=1 \ldots N$. Moreover, for any initial conditions $Q_{i}^{0}>0$, we have that $\lim _{k \rightarrow \infty} Q_{i}(k)=\bar{Q}_{i}$.

PROOF. The proof can be mimicked after the proof of Theorem 1 of [4]. Compared to our case, in Theorem 1 of [4], scalar terms, taking values between zero and one, multiply the matrices $\Sigma_{v_{j}}$ in (A.3). In our case, these scalar terms take the value one, and therefore the result follows. 